\title{
Regarding “Uremic Encephalopathy: MR Imaging Findings and Clinical Correlation"
}

W e thank Kim et $\mathrm{al}^{1}$ for their article on the MR imaging of uremic encephalopathy (UE) in correlation with clinical findings. On a cursory read, the article presents a unique concept: When one reviews an MR imaging study and sees the lentiform fork sign (LFS), consider UE. However, more detailed analysis of the article questions the process used to reach this conclusion.

The LFS was initially described by Kumar and Goyal in 2010. ${ }^{2}$ When they tried to identify an imaging finding specific to UE, a single patient was identified with LFS, uremia, and metabolic acidosis. A subsequently performed literature search found that patients with metabolic acidosis from multiple etiologies also exhibited this sign.

The conclusion of this article sought to shift this association between the LFS and metabolic acidosis to UE. Nine of the 10 patients examined demonstrated the LFS, only 1 of whom demonstrated metabolic acidosis via arterial blood gas. However, only 5 patients in the study had arterial blood gas data, and it is unclear whether the samples were obtained before or after dialysis. The timing of arterial blood gas testing in relation to the MR imaging would be critical for determining whether MR imaging findings had any correlation with metabolic acidosis.

Chronic renal failure (CRF) and metabolic acidosis are strongly associated. ${ }^{3}$ Approximately $80 \%$ of patients with a glomerular filtration rate of $<20$ have metabolic acidosis, as well as most patients on dialysis. This finding suggests that most of the patients were, at some time, acidotic; Kumar and Goyal ${ }^{2}$ reasoned that being acidotic might result in the LFS with normal blood $\mathrm{pH}$. Although it is feasible that routine dialysis might mitigate the degree of metabolic acidosis, the markedly elevated creatinine levels suggest that the patients in this study had not been dialyzed recently. This suggestion, too, is confusing because the article stated that the patients with CRF "regularly received hemodialysis," which should preclude uremia and, therefore, uremic encephalopathy.

Other details briefly mentioned warrant further elaboration. The article states that 1 patient was "subsequently identified and added to the study." There is no discussion of why this patient was not identified initially. Of the 10 patients, 1 had acute renal failure. He may have been excluded if the first search was restricted to chronic renal failure,

http://dx.doi.org/10.3174/ajnr.A5058 but the reasoning behind his eventual inclusion is unclear because he was the only patient not to demonstrate the LFS in the study.

In the "Materials and Methods," the sequences obtained on the 1.5T and 3T magnets are described. There is no mention of FLAIR or gradient recalled-echo/SWI sequences on the $1.5 \mathrm{~T}$ magnet or which patient was scanned on which magnet. This omission has implications for the subsequent "Discussion": When the central variant posterior reversible encephalopathy syndrome (PRES) was mentioned, microhemorrhages on SWI were noted as one of a few key distinguishing features. Of note, the cited article describing central variant PRES found microhemorrhages in 2 of the 4 patients with SWI sequences available, a less strong association than the article implies. ${ }^{4}$

In the "Results," when describing the imaging findings of the 9 patients with chronic renal failure, the authors stated that increased signal on apparent diffusion coefficient maps in the basal ganglia was seen consistent with vasogenic edema. Four of 9 patients had increased signal intensity on diffusion-weighted imaging without restricted diffusion, 2 showed restricted diffusion, and 5 had normal signal on DWI, a total of 11 patients, 2 more than the study population. If all study patients showed the LFS, how could the DWI signal be normal in 5 patients? Could the authors clarify? Because only 4 of 10 patients had follow-up imaging after hemodialysis and the authors did not include the 2 patients with restricted diffusion on the initial study, any conclusion about the reversibility of imaging finding should be viewed with caution. The authors further stated that DWI changes did not correlate with serum creatinine levels between the 2 groups of 4 and 5 patients, but they did not specify what these 2 subgroups were, if restricted diffusion was only seen in 2 patients. Toward the end of the "Discussion," the authors stated that $3 / 9$ patients who underwent DWI showed cytotoxic edema in the globus pallidus; this is very confusing for the readers because the numbers do not match.

If only 2/10 patients had cortical involvement on T2 and FLAIR images and these 2 patients were markedly hypertensive, it is unclear how one can conclude that cortical involvement is a distinct subset of findings in UE.

In the "Discussion," there is mention of diabetes possibly being implicated in the LFS in the presence of UE, a relationship that has been noted previously by Kumar and Goyal. ${ }^{2}$ This article ${ }^{1}$ stated that 7 of the 9 patients with the LFS had diabetes. However, diabetes is the most common cause of renal failure, and all the 
patients with CRF, including 2 with hypertension, demonstrated the LFS. This finding would seem to suggest that all causes of CRF, not just diabetes, are associated.

The article concludes with the statement that the LFS is reliable in the early diagnosis of UE; this conclusion implies some degree of specificity. However, the sensitivity and specificity of the LFS for UE were not determined. It is also unclear how it helped in the "early" diagnosis. Until there is a better understanding of the pathophysiology underlying the imaging findings, it may be more prudent to use the LFS as originally described-to alert clinicians to potential metabolic acidosis.

\section{REFERENCES}

1. Kim DM, Lee IH, Song CJ. Uremic encephalopathy: MR imaging findings and clinical correlation. AJNR Am J Neuroradiol 2016;37: 1604-09 CrossRef Medline
2. Kumar G, Goyal MK. Lentiform fork sign: a unique MRI picture-is metabolic acidosis responsible? Clin Neurol Neurosurg 2010;112: 805-12 CrossRef Medline

3. Kraut JA, Kurtz I. Metabolic acidosis of CKD: diagnosis, clinical characteristics, and treatment. Am J Kidney Dis 2005;45:978-93 CrossRef Medline

4. McKinney AM, Jagadeesan BD, Truwit CL. Central-variant posterior reversible encephalopathy syndrome: brainstem or basal ganglia involvement lacking cortical or subcortical cerebral edema. AJR Am J Roentgenol 2013;201:631-38 CrossRef Medline

(D) N. Das

Department of Radiology and Biomedical Imaging Yale School of Medicine

(1) X. Wu

Yale School of Medicine (1) A. Malhotra Department of Radiology and Biomedical Imaging Yale School of Medicine New Haven, Connecticut 